\title{
Diagnosis and Clinical Course of Unexplained Dysphagia
}

\author{
Jiwoon Yeom, $\mathrm{MD}^{1}$, Young Seop Song, $\mathrm{MD}^{1}$, Won Kyung Lee, $\mathrm{MD}^{1}$, \\ Byung-Mo Oh, MD, PhD ${ }^{1}$, Tai Ryoon Han, MD, $\mathrm{PhD}^{2}$, Han Gil Seo, MD, MS \\ ${ }^{1}$ Department of Rehabilitation Medicine, Seoul National University Hospital, \\ Seoul National University College of Medicine, Seoul; ${ }^{2}$ Gangwon-do Rehabilitation Hospital, Chuncheon, Korea
}

Objective To investigate the final diagnosis of patients with unexplained dysphagia and the clinical and laboratory findings supporting the diagnosis.

Methods We retrospectively analyzed 143 patients with dysphagia of unclear etiology who underwent a videofluoroscopic swallowing study (VFSS). The medical records were reviewed, and patients with a previous history of diseases that could affect swallowing were categorized into a missed group. The remaining patients were divided into an abnormal or normal VFSS group based on the VFSS findings. The clinical course and final diagnosis of each patient were examined.

Results Among the 143 patients, $62(43 \%)$ had a previous history of diseases that could affect swallowing. Of the remaining 81 patients, 58 (72.5\%) had normal VFSS findings and $23(27.5 \%)$ had abnormal VFSS findings. A clear cause of dysphagia was not identified in 9 of the 23 patients. In patients in whom a cause was determined, myopathy was the most common cause $(n=6)$, followed by laryngeal neuropathy $(n=4)$ and drug-induced dysphagia $(n=3)$. The mean ages of the patients in the normal and abnormal VFSS groups differed significantly $(62.52 \pm 15.00$ vs. $76.83 \pm 10.24$ years, respectively; $p<0.001$ by Student $t$-test).

Conclusion Careful history taking and physical examination are the most important approaches for evaluating patients with unexplained swallowing difficulty. Even if VFSS findings are normal in the pharyngeal phase, some patients may need additional examinations. Electrodiagnostic studies and laboratory tests should be considered for patients with abnormal VFSS findings.

Keywords Deglutition disorders, Diagnosis, Etiology, Videofluoroscopy

Received June 2, 2015; Accepted August 7, 2015

Corresponding author: Han Gil Seo

Department of Rehabilitation Medicine, Seoul National University Hospital, Seoul National University College of Medicine, 101 Daehak-ro, Jongno-gu, Seoul 03080, Korea

Tel: +82-2-2072-1659, Fax: +82-2-743-7473, E-mail: hgseo80@gmail.com

(c) This is an open-access article distributed under the terms of the Creative Commons Attribution Non-Commercial License (http://creativecommons. org/licenses/by-nc/4.0) which permits unrestricted noncommercial use, distribution, and reproduction in any medium, provided the original work is properly cited.

Copyright $\odot 2016$ by Korean Academy of Rehabilitation Medicine

\section{INTRODUCTION}

Dysphagia is generally defined as any kind of swallowing difficulty [1]. The prevalence of dysphagia in the South Korean general population aged $>65$ years is reported as $33.7 \%$ [2]. This condition is commonly caused by various diseases, including central nervous system (CNS) disorders, neurodegenerative diseases, peripheral nervous system disorders, neuromuscular junction disorders, myopathies, local anatomical lesions, and psycho- 
genic disorders [3]. Dysphagia can lead to pneumonia, malnutrition, dehydration, and increased mortality [4]. For proper diagnostic evaluation of dysphagia, careful history taking is the first step [5]. The next steps are physical and neurologic examinations and appropriate instrumental studies, such as a videofluoroscopic swallowing study (VFSS).

Patients with dysphagia-related symptoms without definite etiology are often encountered, and diagnostic evaluation and differential diagnosis can be challenging owing to varying dysphagia etiologies. Investigation of patient characteristics with unexplained dysphagia may provide useful information to clinicians for approaching the final diagnosis in these patients.

The purpose of this study was to investigate the final diagnosis of patients with unexplained dysphagia and the clinical and laboratory findings supporting the diagnosis.

\section{MATERIALS AND METHODS}

\section{Subjects}

A retrospective chart review was performed in a university-affiliated tertiary care hospital. Between January 1 , 2007 and December 31, 2012, 6,729 patients underwent VFSS. Among these patients, swallowing difficulty with unexplained etiology at the first medical examination during inpatient consultation and in the outpatient clinic before VFSS was identified in 143 patients. These patients with unknown etiology were included in the present study. The demographic characteristics of the patients, including clinical data and clinical dysphagia scale (CDS) scores, were presented in Table 1. The CDS is a dysphagia rating scale that includes 8 rating items (lesion location, tracheostomy, history of aspiration, lip sealing, chewing and mastication, tongue protrusion, laryngeal elevation, and reflex coughing) [6]. The mean age of the patients was 69.5 years at the time of VFSS.

\section{Medical chart review}

Through medical chart review, we extracted data on age, sex, diagnosis before VFSS, department from which referral for VFSS was made, current diet, CDS score, recommended diet in the current VFSS, videofluoroscopic dysphagia scale score, American Speech-LanguageHearing Association National Outcome Measurement System (ASHA NOMS) swallowing scale, course of hos-
Table 1. Demographic characteristics $(n=143)$

\begin{tabular}{|lc|}
\hline \multicolumn{1}{|c}{ Characteristic } & Value \\
\hline Age (yr) & $69.5 \pm 13.5$ \\
\hline Sex & \\
\hline Male & $86(60.1)$ \\
\hline Female & $57(39.9)$ \\
\hline Duration of dysphagia & $73(51.0)$ \\
\hline$<3$ mo & $17(11.9)$ \\
\hline 3 mo to 1 yr & $40(28.0)$ \\
\hline$>1$ yr & $13(9.1)$ \\
\hline Unclear & $6(4.2)$ \\
\hline Clinical dysphagia scale & $53(37.1)$ \\
\hline Tracheostomy & \\
\hline Aspiration symptom & $22(15.4)$ \\
\hline Lip sealing & $2(1.4)$ \\
\hline Inadequate & \\
\hline None & $25(17.5)$ \\
\hline Chewing and mastication & $2(1.4)$ \\
\hline Inadequate & \\
\hline None & $24(16.8)$ \\
\hline Tongue protrusion & $4(2.8)$ \\
\hline Inadequate & $58(40.6)$ \\
\hline None & $3(2.1)$ \\
\hline Laryngeal elevation & $26(18.2)$ \\
\hline Inadequate & \\
\hline None & \\
\hline Reflex coughing & \\
\hline
\end{tabular}

Values are presented as mean \pm standard deviation or number (\%).

pital care, and final diagnosis. Patients with a previous history of diseases that could affect swallowing at the time of the study were classified into a missed group. The remaining patients were further divided into the following 2 groups based on the VFSS findings: a normal VFSS group and abnormal VFSS group. An abnormal VFSS finding was considered if $\geq 1$ of the following traits were noted: 1) requirement of diet modification after VFSS, 2) definite aspiration, 3) pharyngeal delay time over 2 seconds, 4) incomplete laryngeal closure, 5) lack of epiglottic folding, 6) incomplete velopharyngeal closure and 7) impaired upper esophageal sphincter opening. The clinical course and final diagnosis of each patient were examined in both groups with a thorough review of the medical records. The study protocol was approved by the 
Institutional Review Board of Seoul National University Hospital (IRB No. 1412-006-629).

\section{Statistical analysis}

The causes of dysphagia were assessed in the study patients. The mean ages of the patients in the normal and abnormal VFSS groups were compared using the Student t-test. PASW Statistics software for Windows (ver. 18, SPSS Inc., Chicago, IL, USA) was used for the statistical analysis. A p-value $<0.05$ was considered significant.

\section{RESULTS}

Fig. 1 showed the flow chart for grouping of the study patients. Of the 143 patients, $62(43.4 \%)$ had a previous history of diseases that could affect swallowing before a VFSS and 81 (56.6\%) did not have such a history. Problems of the CNS (57/62, 91.9\%), such as stroke, Parkinsonism, dementia, brain tumor, and traumatic brain injury, were the major causes of swallowing difficulty in patients who had a dysphagia history. Additionally, 9 patients had a history of receiving intensive care treatment or intubation suggestive of possible post-extubation dys- phagia, 5 had been diagnosed with head and neck cancer or esophageal cancer, and 4 had lower cranial neuropathy. Moreover, 1 patient each had a history of drug intoxication, steroid myopathy, and myasthenia gravis. In this missed group, 16 patients had more than 1 etiology (Table 2). Among the 81 patients who were analyzed further, 58 (72.5\%) had normal VFSS findings, and no pathology was identified in more than half of these 58 patients $(33 / 58$, $57 \%$ ). Reflux diseases, such as laryngopharyngeal reflux and gastroesophageal reflux, were the most common causes of dysphagia $(11 / 58,19.0 \%)$, followed by globus hystericus (5/58, 8.6\%) and laryngospasm (4/58, 6.9\%). Moreover, 1 patient each had amyotrophic lateral sclerosis, achalasia, bronchoesophageal fistula, cricopharyngeal bar, laryngeal hyperirritability, hyperemesis, and phagophobia in a follow-up study. In this normal VFSS group, 2 patients had more than 1 etiology (Table 3 ).

Abnormal VFSS findings were observed in 23 patients $(23 / 80,28.8 \%)$, and no clear cause of dysphagia was identified in 9 patients. Among patients with known cause of dysphagia, myopathy was the most common cause $(6 / 23$, $26.1 \%)$. Among these patients, 3 were finally diagnosed with oculopharyngeal muscular dystrophy (OPMD) and
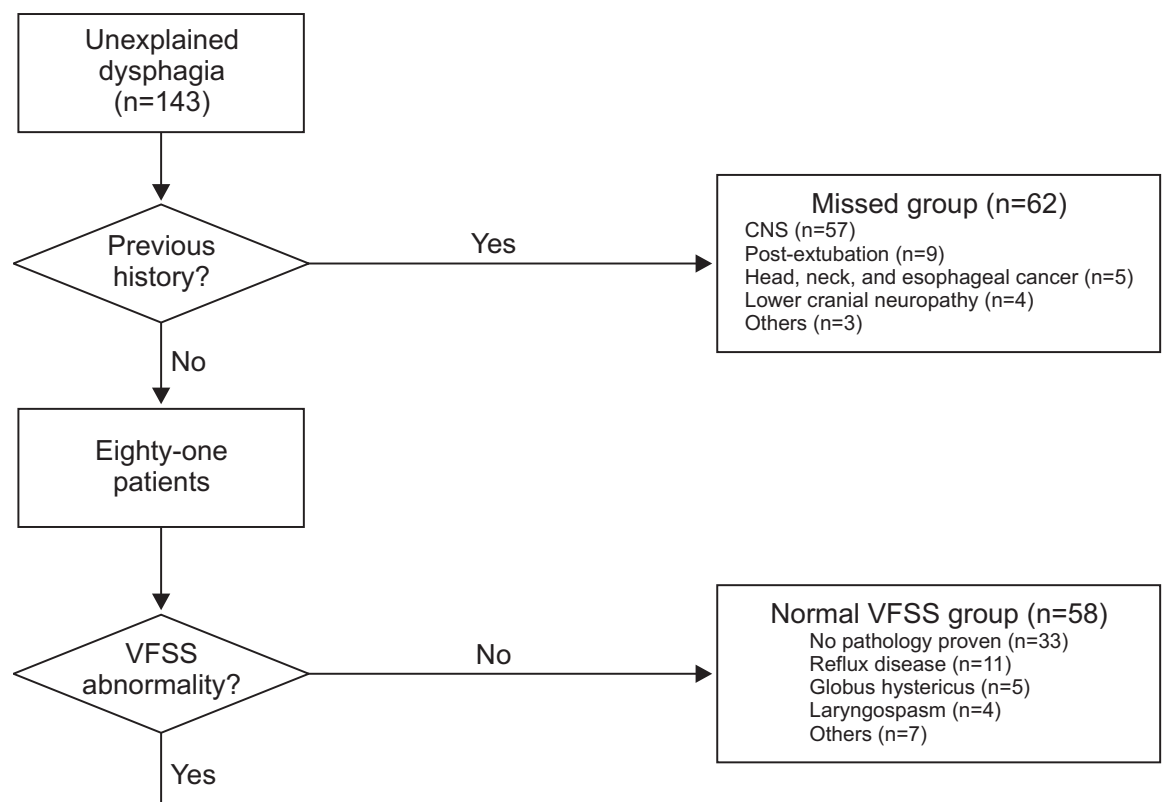

Abnormal VFSS group $(n=23)$ Myopathy $(n=6)$

Laryngeal neuropathy $(n=4)$

Drug induced dysphagia $(n=3)$

Thyrotoxicosis $(\mathrm{n}=1)$

Unknown $(n=9)$
Fig. 1. A total of $62(43 \%)$ patients had etiologies that could cause swallowing difficulty before undergoing a videofluoroscopic swallowing study (VFSS), and most of these patients had a cerebrovascular accident (CVA) history. No pathology was proven in $33(23 \%)$ patients with normal VFSS findings. VFSS abnormalities were confirmed in $23(16 \%)$ patients. CNS, central nervous system. 
Table 2. Missed etiologies at the first visit (62 patients; 16 had more than 1 etiology)

\begin{tabular}{lc}
\hline \multicolumn{1}{c}{ Etiology } & No. \\
\hline Central nervous system problems & 57 \\
Post-extubation dysphagia & 9 \\
Head, neck, and esophageal malignancies & 5 \\
Lower cranial neuropathy & 4 \\
Drug intoxication & 1 \\
Steroid myopathy & 1 \\
Myasthenia gravis & 1 \\
\hline
\end{tabular}

Table 3. Etiologies in patients with normal video fluoroscopic swallowing study findings (58 patients; 2 had more than 1 etiology)

\begin{tabular}{|lc|}
\hline \multicolumn{1}{|c}{ Etiology (examination) } & No. \\
\hline No pathology proven & 33 \\
\hline Reflux disease & 11 \\
\hline Globus hystericus & 5 \\
\hline Laryngospasm & 4 \\
\hline ALS (electrodiagnosis) & 1 \\
\hline Achalasia (esophagography, & 1 \\
\hline esophagogastroduodenoscopy) & \\
\hline Bronchoesophageal fistula (Neck CT) & 1 \\
\hline Cricopharyngeal bar & 1 \\
\hline Laryngeal hyperirritability & 1 \\
\hline Hyperemesis & 1 \\
\hline Phagophobia & 1 \\
\hline
\end{tabular}

ALS, amyotrophic lateral sclerosis; CT, computed tomography.

the others were diagnosed with generalized myopathies. All myopathy cases were confirmed using electromyography (EMG), and 2 cases of OPMD were confirmed with PABPN1 gene studies. Laryngeal neuropathy was diagnosed in 4 patients using laryngoscopy and laryngeal EMG. Additionally, 3 patients had drug-induced dysphagia, and thyrotoxicosis was the cause of dysphagia in 1 patient (Table 4).

The mean ages of the patients in the normal and abnormal VFSS groups differed significantly $(62.52 \pm 15.00$ vs. $76.83 \pm 10.24$ years, respectively; $\mathrm{p}<0.001$ by Student ttest).
Table 4. Etiologies in patients with abnormal videofluoroscopic swallowing study findings (total 23 patients)

\begin{tabular}{lc}
\hline \multicolumn{1}{c}{ Etiology (examination) } & No. \\
\hline Myopathy (electromyography, gene study) & 6 \\
$\begin{array}{l}\text { Laryngeal neuropathy (laryngeal } \\
\text { electromyography) }\end{array}$ & 4 \\
Unknown & 9 \\
Drug-induced dysphagia & 3 \\
Thyrotoxicosis (thyroid function test) & 1 \\
\hline
\end{tabular}

\section{DISCUSSION}

This study identified dysphagia etiologies in patients with unknown etiologies at initial visit. All patients were classified according to the final diagnosis. However, the identified condition might not be the direct cause of dysphagia. In order to reduce the error, the medical records and clinical course of each patient were analyzed in detail. Before performing a VFSS, $43 \%$ of the patients had established etiologies that could cause swallowing difficulty. Among the remaining $57 \%$ of the patients, the cause of dysphagia was not identified in $41 \%$ of those with normal VFSS findings. Additionally, $14 \%$ were diagnosed with reflux disease, and $27 \%$ had confirmed VFSS abnormalities. After VFSS, myopathy and laryngeal neuropathy were finally diagnosed through further examinations.

In almost half of the patients included in the study, previous conditions affecting swallowing that could explain the cause of the present dysphagia were overlooked at the initial visit. A previous study indicated that good history taking can help in the determination of the anatomical site and the likely cause of dysphagia in $80 \%$ of cases [3]. Additionally, the importance of history taking and physical examination was demonstrated in an earlier study [5].

In the present study, 33 patients had no definite abnormal findings in a VFSS and had not been diagnosed with a condition affecting swallowing. In the clinical course of this group, no findings indicated whether the symptoms persisted or worsened.

Even if the VFSS findings are normal, the possibility of other causes of dysphagia should be considered. In the present study, diseases that could lead to dysphagia were later identified in $43 \%$ of the patients with normal VFSS findings. These results demonstrated that esophageal disorders as well as oropharyngeal disorders are com- 
mon causes of dysphagia.

Reflux diseases $(n=11)$, including gastroesophageal reflux, reflux esophagitis, and laryngopharyngeal reflux, were also common. Patients with reflux diseases often have aspiration pneumonia, that is misdiagnosed as oropharyngeal dysphagia [7].

The features of esophageal dysmotility are dysphagia (for solids and liquids), chest pain, and regurgitation. The abrupt onset, duration, or rate of progression of dysphagia can help in the differential diagnosis. In general, patients who have motility disorders, such as achalasia and diffuse esophageal spasm, experience dysphagia for liquids and solids, whereas patients who have structural disorders, such as a tumor and hiatal hernia, experience dysphagia for solids only. Patients with globus hystericus may experience the feeling of difficulty in swallowing. However, globus hystericus arguably does not belong in the category of dysphagia [3]. In fact, many globus hystericus cases were misdiagnosed owing to failure to fully rule out other diseases [8]. However, generally, dysphagia is defined as difficulty moving food from the mouth to the stomach or any kind of swallowing difficulty. Accordingly, patients with globus hystericus were included in our study. Therefore, careful history taking is vital for the evaluation of dysphagia. The purpose of history taking in patients with dysphagia is to determine whether dysphagia is truly present, determine whether the problem is located in the esophagus or pharynx, and distinguish a structural abnormality from a motor disorder [5].

There were some interesting findings in the normal VFSS group that provides important information. First, in patients with a slow progressive disease, a VFSS performed in the early stage may show normal findings. Dysphagia in patients with amyotrophic lateral sclerosis (ALS) reportedly affects the oral and pharyngeal regions [9]. Moreover, a delay in triggering the swallowing reflex has been reported [10]. In the VFSS findings of 1 of our patients, premature bolus loss and vallecular residue were moderate, and definite aspiration was not observed. However, the patient felt sustained discomfort and was eventually diagnosed with ALS through a workup that included EMG. Careful follow-up is necessary for these patients [11]. Second, post-VFSS posteroanterior chest examination as well as VFSS is important. These can provide clues about esophageal disorders, and the diagnosis can be confirmed after further examination, including esophagogastroduodenoscopy and esophagography. Third, although VFSS is a gold standard examination for dysphagia, it is hard to fully evaluate esophageal dysphagia. In particular, bronchoesophageal fistulae are often diagnosed with radiocontrast studies of the esophagus or incidentally during operations. These patients complain of chronic cough associated with drinking [12]. Therefore, clinicians should always be aware that patients can experience swallowing difficulty because of various conditions. Despite normal findings on a VFSS, patient follow-up is necessary $s$ if discomfort persists.

Abnormal findings were identified in 23 of 81 patients, and myopathy $(n=6)$ was the most common disease in this group. All patients with myopathy presented with dysphagia as the initial symptom. Oh et al. [13] reported that dysphagia was a presenting symptom in $21 \%$ of patients with inflammatory myopathy. In our study, higher amounts of vallecular and pyriform sinus residues and poorer relaxation of the upper esophageal sphincter were noted in myopathy patients than in other patients on performing a VFSS. However, these differences were not statistically significant, perhaps because of the small sample size. Laryngeal neuropathy was confirmed with a laryngeal EMG in 4 patients, and most of these patients had a history of hoarseness. The role of laryngeal EMG in the evaluation of voice problems is well known [14].

The 3 patients with drug-induced dysphagia showed polypharmacy. Anticholinergics cause dysphagia by affecting the smooth and striated muscles, such as those in the esophagus [15]. In our study, 3 patients were taking anticholinergics prior to experiencing dysphagia. In addition to these patients, many other patients had taken drugs that can cause xerostomia, drowsiness, and confusion. In earlier studies, these drugs are reported to possibly induce dysphagia [16], and the above-mentioned symptoms are likely to affect swallowing. However, these patients were included in the unknown group because of the relatively low correlation of these drugs with dysphagia. A clear cause of dysphagia was not identified in 9 patients. In 3 patients, a neuromuscular disorder was suspected; however, no abnormalities were noted on EMG. Among these 3 patients, 2 were lost to follow-up and 1 underwent follow-up VFSSs 7 times, with no change in the clinical course and ASHA NOMS scale indicating no improvement in swallowing function. The other patients in this unknown group could be in a deconditioning 
state or have a complex medical condition. In 2 of these patients, swallowing function showed improvement at a follow-up VFSS with recovery from underlying diseases such as pneumonia (ASHA NOMS scale from 4 to 6) and hyperglycemic hyperosmolar syndrome (ASHA NOMS scale from 2 to 5); however, the other patients $(n=4)$ did not show any improvement in the swallowing function or did not undergo follow-up examination owing to death or other factors. Interestingly, 1 patient, a 77-year-old man, experienced dysphagia as a manifestation of thyrotoxicosis. Compared to the incidence of dysphagia caused by myopathy, dysphagia caused by thyrotoxicosis is uncommon. Dysphagia caused by thyrotoxicosis tends to resolve after treatment of the thyrotoxicosis [17]. Therefore, after excluding a diagnosis with a high potential, endocrinologic screening could be considered.

When the study was started, the incidence of age-related dysphagia appeared to be high. While direct causes of age-related dysphagia were not identified, several causes could be considered. First, small infarctions not detected on magnetic resonance imaging (MRI) could be a cause. A previous study by Buchholz [5] proposed this possibility, but was discussed in terms of low-resolution MRI. Because detection using high-resolution MRI might be greater, these patients could not be regarded with presbyphagia. Second, elderly people tend to take many medicines because of various diseases, i.e., 'polypharmacy,' and these patients are likely to experience druginduced dysphagia. Third, elderly people with a relatively poor immune function and a small amount of aspiration can experience high pneumonia morbidity. Pneumoniarelated deterioration is shown to also cause dysphagia alone [18]. In fact, little is known about presbyphagia, and healthy swallowing is unclear in the elderly [19]. In the present study, age differed significantly between the normal and abnormal VFSS groups. The mean age of the patents in the abnormal VFSS group was 14 years older than that of the patients in the normal VFSS group. Therefore, dysphagia should not be considered to result from the aging process, and clinicians should try to identify its true cause.

The present study had several limitations. First, this was a retrospective and single-center study in a universityaffiliated tertiary care hospital. Therefore, there is a possibility of selection bias. For this reason, conditions, such as cervical anterior osteophyte, which can be eas- ily confirmed with a lateral cervical spine radiography or VFSS, were not found in our study. Second, patients were classified according to the final diagnosis that could induce dysphagia. However, there is a possibility of no causal relationship between dysphagia and diagnosis, in particular, in the missed group. Sixteen patients with more than 1 etiology might suggest no definite causal relationship between dysphagia and diagnosis. However, if patients have an underlying disease possibly causing dysphagia, it is inappropriate to classify them into unexplained dysphagia. Third, only patients in whom the cause of dysphagia could be determined at the first visit were included in the investigation. As mentioned previously, even if a history of a condition is noted (for example stroke), other causes should not be overlooked. Therefore, a prospective study is needed to avoid the possibility of selection bias in the future.

In summary, careful history taking and physical examination are the most important approaches in the evaluation of patients with unexplained swallowing difficulty. Even if VFSS findings are normal in the pharyngeal phase, some patients may need additional gastroenterological and otorhinolaryngological examinations. Electrodiagnostic studies should be considered for patients with abnormal VFSS findings. Laboratory tests, such as thyroid function, creatine kinase, and lactate dehydrogenase tests, may be useful in the screening of these patients.

\section{CONFLICT OF INTEREST}

No potential conflict of interest relevant to this article was reported.

\section{REFERENCES}

1. Martino R, Pron G, Diamant N. Screening for oropharyngeal dysphagia in stroke: insufficient evidence for guidelines. Dysphagia 2000;15:19-30.

2. Yang EJ, Kim MH, Lim JY, Paik NJ. Oropharyngeal Dysphagia in a community-based elderly cohort: the Korean longitudinal study on health and aging. J Korean Med Sci 2013;28:1534-9.

3. Cook IJ. Diagnostic evaluation of dysphagia. Nat Clin Pract Gastroenterol Hepatol 2008;5:393-403.

4. Cabre M, Serra-Prat M, Palomera E, Almirall J, Pallares R, Clave P. Prevalence and prognostic implica- 
tions of dysphagia in elderly patients with pneumonia. Age Ageing 2010;39:39-45.

5. Buchholz DW. Neurogenic dysphagia: what is the cause when the cause is not obvious? Dysphagia 1994;9:245-55.

6. Han TR, Paik NJ, Park JW. The clinical functional scale for dysphagia in stroke patients. Korean J Stroke 2001;3:153-7.

7. Tauber S, Gross M, Issing WJ. Association of laryngopharyngeal symptoms with gastroesophageal reflux disease. Laryngoscope 2002;112:879-86.

8. Ravich WJ, Wilson RS, Jones B, Donner MW. Psychogenic dysphagia and globus: reevaluation of 23 patients. Dysphagia 1989;4:35-8.

9. Kawai S, Tsukuda M, Mochimatsu I, Enomoto H, Kagesato $\mathrm{Y}$, Hirose $\mathrm{H}$, et al. A study of the early stage of Dysphagia in amyotrophic lateral sclerosis. Dysphagia 2003;18:1-8.

10. Ertekin C, Aydogdu I, Yuceyar N, Kiylioglu N, Tarlaci S, Uludag B. Pathophysiological mechanisms of oropharyngeal dysphagia in amyotrophic lateral sclerosis. Brain 2000;123(Pt 1):125-40.

11. Kim S, Kang SW, Choi W, Park JH, Lee Y, Yu SJ. Survey on the diagnostic process of amyotrophic lateral sclerosis. J Korean Acad Rehabil Med 2011;35:110-4.
12. Ford MA, Mueller PS, Morgenthaler TI. Bronchoesophageal fistula due to broncholithiasis: a case series. Respir Med 2005;99:830-5.

13. Oh TH, Brumfield KA, Hoskin TL, Stolp KA, Murray JA, Bassford JR. Dysphagia in inflammatory myopathy: clinical characteristics, treatment strategies, and outcome in 62 patients. Mayo Clin Proc 2007;82:441-7.

14. Woo P. Laryngeal electromyography is a cost-effective clinically useful tool in the evaluation of vocal fold function. Arch Otolaryngol Head Neck Surg 1998;124:472-5.

15. O'Neill JL, Remington TL. Drug-induced esophageal injuries and dysphagia. Ann Pharmacother 2003;37:1675-84.

16. Al-Shehri AM. Drug-induced dysphagia. Ann Saudi Med 2003;23:249-53.

17. Chiu WY, Yang CC, Huang IC, Huang TS. Dysphagia as a manifestation of thyrotoxicosis: report of three cases and literature review. Dysphagia 2004;19:120-4.

18. Marik PE, Kaplan D. Aspiration pneumonia and dysphagia in the elderly. Chest 2003;124:328-36.

19. Leslie P, Drinnan MJ, Ford GA, Wilson JA. Swallow respiratory patterns and aging: presbyphagia or dysphagia? J Gerontol A Biol Sci Med Sci 2005;60:391-5. 